\title{
Single-port cholecystectomy with the TransEnterix SPIDER: simple and safe
}

\author{
Aurora D. Pryor $\cdot$ John R. Tushar $\cdot$ Louis R. DiBernardo
}

Received: 19 April 2009/Accepted: 25 August 2009/Published online: 16 September 2009

(C) The Author(s) 2009. This article is published with open access at Springerlink.com

\begin{abstract}
Introduction Single-port or single-incision cholecystectomy with current rigid laparoscopic devices is limited by in-line visualization, restricting the ability to approach the surgical site with proper angles and instrumentation. A single-port access system with articulating arms and strong instrumentation should minimize these issues. The TransEnterix system may facilitate safe and straightforward single-port surgery.

Methods The TransEnterix single-port surgery system was used in both survival and nonsurvival porcine laparoscopic cholecystectomies under animal use committee approval. Nonsurvival procedures compared four standard laparoscopic with four single-port cholecystectomies from a histologic perspective. Five single-port swine laparoscopic cholecystectomy procedures were completed in sterile conditions, and all animals survived for 1 week postoperatively. Standard surgical clips were used for both cystic duct and artery ligation. At sacrifice, both gross and microscopic histology were obtained for assessment of surgical complications.

Results All cholecystectomies were successfully completed with the TransEnterix single-port system. Operative time for the survival procedures averaged 39.4 (range 1866) min. Histology of the acute specimens showed less inflammation at the single-port site compared with the
\end{abstract}

\author{
A. D. Pryor $(\bowtie)$ \\ Department of Surgery, Duke University, Durham, NC, USA \\ e-mail: pryor002@mc.duke.edu \\ J. R. Tushar \\ TransEnterix, Inc., Research Triangle Park, NC, USA \\ L. R. DiBernardo \\ Department of Pathology, Duke University, Durham, NC, USA
}

trocar sites from the standard cholecystectomy. At sacrifice, no complications were identified.

Conclusions The TransEnterix system is safe and straightforward for completing single-port cholecystectomy in this limited porcine series. Port site inflammation is reduced compared with standard laparoscopic trocars.

Keywords Single Port - Cholecystectomy · Laparoscopic · Minimally Invasive

Single-port surgery (SPS), often referred to as single-site laparoscopic surgery, is characterized by making a single incision through the umbilicus to enter the abdominal cavity and perform surgery [1]. SPS has emerged as a new approach, and potentially an alternative to conventional multi-port laparoscopy [2-6]. Recent advances in laparoscopic surgery include single-port access devices that facilitate a single-incision and single-site ports, which include multiple channels to accommodate multiple surgical instruments through one access site [4, 5, 7]. These channels provide a pathway to the abdomen, thus eliminating the requirement for multiple incisions and multiple trocars. SPS has the potential to disrupt the current laparoscopic surgical field, similar to how laparoscopy challenged open surgery 20 years ago.

Although the concept of SPS has evolved during the past 3 years, no ideal system is currently available. Current techniques use rigid instruments through low-profile trocars and certain novel instruments have articulating distal portions; however, surgeons often operate cross-handed to adjust and compensate for instruments crossing, in a scissor-like manner, within the abdominal cavity. In addition, current single-port techniques pose challenges of obtaining critical views, visual orientation, and logical manipulation 
of the surgical instruments. These approaches fail to provide the surgeon with the necessary angles for ideal orientation of surgical instruments and safe identification of critical structures [8]. A practical single-port system, which could effectively replicate multi-port laparoscopy and potentially replace it, will need to enable triangulation and have effective retraction capabilities.

Other concerns with single-site techniques include issues relating to the access site where excessive tissue trauma may occur due to a larger port site access, carrying the added risk of infection, delayed healing, and herniation. Multiple fascial incisions through one access site might lead to weakened fascia or seroma formation from the significant dissection required. SPS with the Single-Port Instrument Delivery Extended Research (SPIDER developed by TransEnterix, Inc., Research Triangle Park, NC) may improve single-site surgery. The device is smaller in diameter than other single-site systems and, therefore, can minimize fascial insult. It also offers articulation and angles that often are lacking with single-site techniques.

We hypothesized that performing laparoscopic singlesite surgery with the SPIDER system would provide angles and exposure to the surgical site at least comparable to standard laparoscopy. We proposed that by moving instrument manipulation past the level of the skin and fascia that local wound inflammation would be minimized compared with standard laparoscopy.

\section{Methods}

We used the TransEnterix SPIDER single-port surgery system to complete laparoscopic cholecystectomies in swine with the approval of the animal use committee. The SPIDER is designed to allow multiple surgical instruments to be advanced and manipulated through a single port (Fig. 1). The single port, or cannula, encapsulates four working channels: two static, and two flexible, thereby eliminating the need for multiple abdominal punctures, and requiring only one single umbilical incision. Once the SPIDER is advanced into the abdominal cavity, the flexible instrument delivery tubes are deployed and used to guide surgical instruments to the surgical site. With the SPIDER system, the operative field visuals and instrument orientation (triangulation) replicate current laparoscopic techniques, and critical views are maintained throughout the surgical procedures (Fig. 2). The outer diameter of the system is only $18 \mathrm{~mm}$, which allows access through a standard open access incision.

The SPIDER is a sterile and disposable device and is used to facilitate the movements of multiple instruments during laparoscopic surgical procedures, performing the following functions.

After an open cut down incision, the multichannel cannula is inserted through a small abdominal incision. The channels are deployed allowing laparoscopic instruments to pass through each channel into the abdomen to perform laparoscopic surgery. Pneumoperitoneum is maintained through the device during the surgical procedure.

The single-port access device contains the following components:

- A retractable sheath that covers the distal end of the SPIDER, which includes the main body port (cannula) and the extended reach Instrument Delivery Tubes (IDTs). The sheath protects internal tissues as the cannula is advanced through the abdominal wall and is

\section{SPIDER DEVICE}

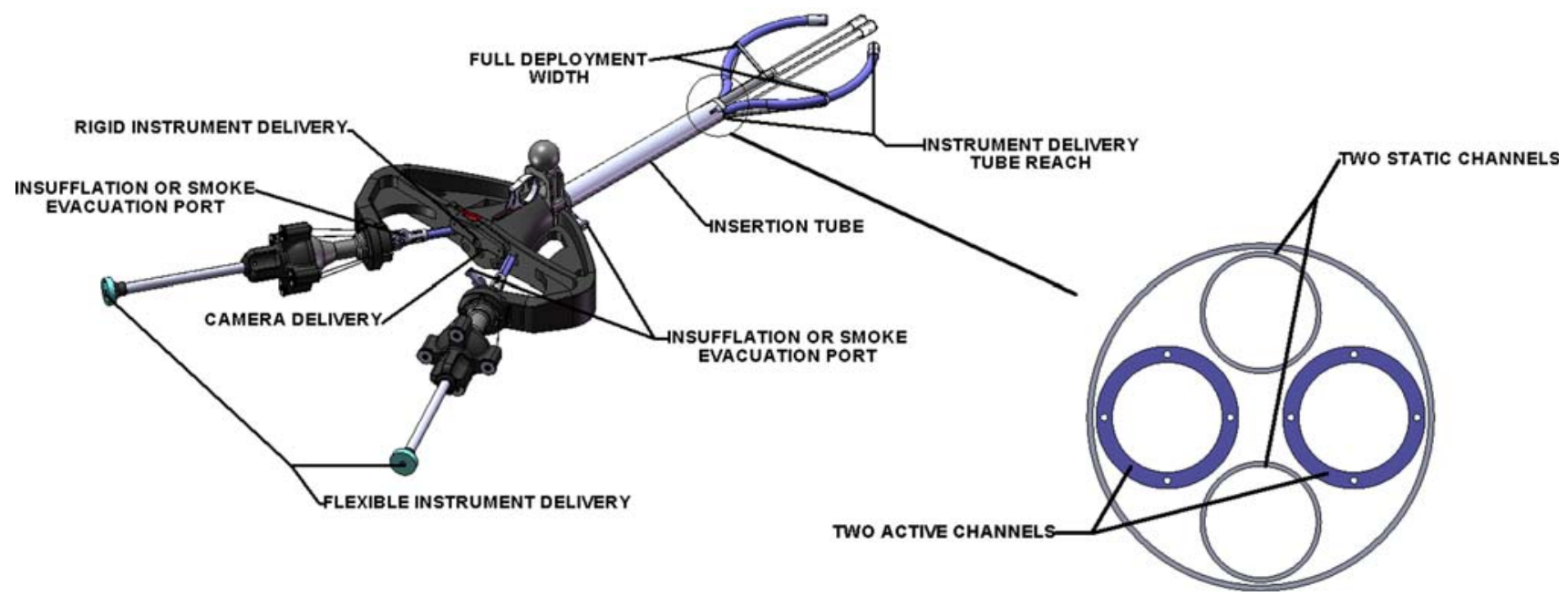

Fig. 1 TransEnterix SPIDER Single Port Surgery Device and cross-sectional view of delivery tube depicting four working channels 


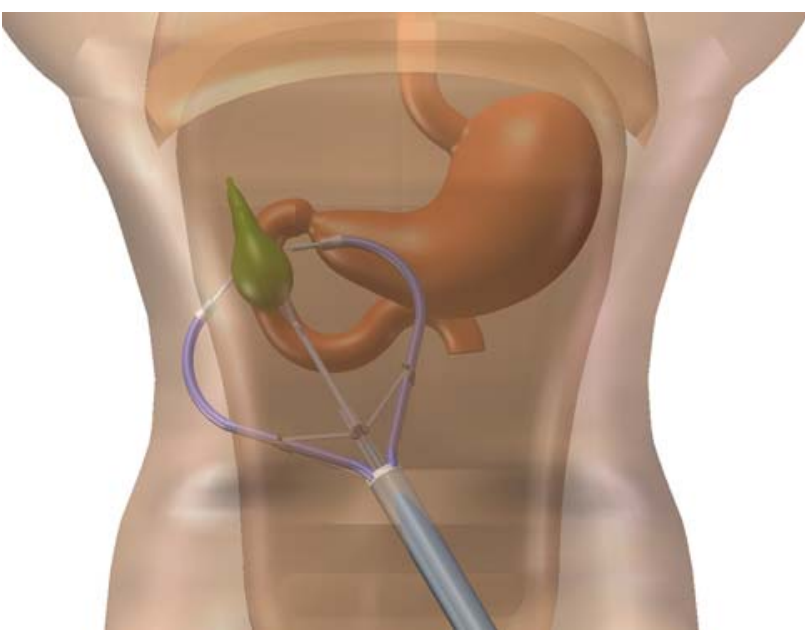

Fig. 2 SPIDER deployed for laparoscopic cholecystectomy

pulled back once the cannula is in position. The sheath also can be advanced at the conclusion of the procedure to facilitate specimen extraction.

- Four working channels

Two channels, known as IDTs, are positioned laterally to include extended lumens to facilitate manipulation of flexible surgical instruments, enabling control of the instruments over extended distances. The IDTs are flexible and allow for $x, y$, and $z$ motion for a multidirectional approach into and throughout the surgical field, mimicking the approach of standard laparoscopic surgery.

The two flexible IDTs are actuated by a gimbal system at the proximal end, which provides 360 degrees of freedom at the distal end.

Two rigid channels, superiorly and inferiorly, can accommodate an endoscope or any of the shelf rigid surgical instruments with a dimension of $<6 \mathrm{~mm}$.

- Three distinct ports for insufflation or smoke evacuation.

- Valves that maintain the pneumoperitoneum established for the surgical procedures.

The SPIDER device also includes a support arm accessory to mount and stabilize the device.

The Instrument Delivery Tubes perform the function of cannulas to guide and maneuver surgical instrumentation. The flexible IDTs provide $x, y$, and $z$ motion to allow for a multidirectional approach of the surgical field, mimicking the approach of standard laparoscopic surgery. The SPIDER system has been successfully used in more than 118 preclinical tests to date.

Standard four-port laparoscopic cholecystectomy was acutely compared with single-site SPIDER cholecystectomy in four swine each. At the completion of the procedures, all abdomens were widely opened and examined for bruising or lacerations, looking for unexpected evidence of injury to the peritoneal surfaces of the abdominal cavity caused by the platform or port during cholecystectomy. In addition, the operative fields were examined for evidence of hepatic trauma, bile leak or biloma, hematoma, or abscess formation. The trocar sites were widely excised and sent for histologic evaluation. After fixation in $10 \%$ buffered formalin, the incisions were serially sectioned in a plane perpendicular to the trocar site/ incision. Gross and microscopic examinations were performed. At least five sections from each incision were examined, including superior and inferior margins, mid superior, mid incision, and mid inferior incision, thus providing a thorough representation of the entire incision in case tissue compression/injury was not uniform across the entire incision. All sections were stained with hematoxylin and eosin as well as Masson trichrome stains. We compared full-thickness sections of tissue from the sites for evidence of tissue necrosis, inflammation, and hemorrhage. Compression necrosis and abrasions were assessed at the level of skin or epidermis, abdominal wall adipose tissue, rectus musculature, and linea alba and parietal peritoneum.

Five SPIDER procedures were subsequently completed under sterile conditions and all animals survived for 1 week postoperatively. Standard surgical clips were used for both cystic duct and artery ligation. At sacrifice, both gross and microscopic histology were obtained for assessment of surgical complications. We again evaluated the local soft tissue trauma from the skin to the deeper adipose tissue, including the assessment of the integrity of the fascia to determine whether there were any potential risks with wound closure. The abdominal wall was assessed for progression of any tissue necrosis to determine whether injury occurred that was not apparent in the acutely sacrificed study device examinations. Healing was assessed for extent and type of inflammation and compared with well-established knowledge of expected 7-day wound healing. Additionally, we evaluated deep tissue reaction at the operative field of the gall bladder fossa and right upper quadrant of the peritoneal cavity to include the extent of inflammation or hemorrhage, if present, and any evidence of bile staining or leakage at the port site during removal of the gall bladder. Serial sections of the gall bladder bed were performed for gross and microscopic examination. Wound healing in standard laparoscopic approach is well understood and did not warrant sacrifice of additional animals.

\section{Results}

All four single-port and four acute cholecystectomies were successfully completed without apparent complications. 
For the acute studies, on gross examination there was minimal inflammation or hemorrhage and no real apparent trauma at the level of skin or epidermis with the single-port sections. No operative site complications were noted. In the standard multi-port sections, the skin demonstrated increased epidermal sloughage and focal hemorrhage compared with the single-port device incision. There also was apparent deep tissue injury with mildly increased hemorrhage in the local abdominal muscles and tissue planes. Many of the ports did not pass directly perpendicular to the skin but skewed along deeper tissue planes before entering the peritoneal cavity. This was associated with creation of potential spaces and increased hemorrhage along the fascial planes. The site of entry into the peritoneal cavity was frequently not within the fascial connective tissue but through abdominal wall musculature. All study device sites were midline and within the linea alba.

Microscopic examination confirmed that in the singleport cross sections there was minimal tissue trauma at the skin level. Tissue necrosis was limited to several cell layers thickness at the surface of the incision. Minimal compression injury was noted. In fact, there appeared to be more local tissue injury from cautery effect than compression trauma from the larger study device port. There was no significant sloughing, no compression injury, and more importantly in regards to wound healing, the adipose tissue of the abdominal wall remained viable and the fascia was without injury as well. In these acute studies, there was minimal acute inflammation and very little abdominal wall hemorrhage.

Standard multiport laparoscopy cross section analysis confirmed that there was direct injury to abdominal muscles, with several of the ports placed through the muscles versus through connective tissue planes. Also, these ports were shown to track through tissue planes instead of passing directly in a perpendicular fashion through the abdominal wall, this was associated with creating potential spaces as well as a mild increase in hemorrhage within the deeper tissue.

Under microscopic examination, the grossly appreciated differences were confirmed. There was an increase in compression trauma necrosis with the 5-mm port, generally at the skin level consistent with placement of the port through a smaller stab wound compared with an open incision. The muscle and tissue plane splitting along with increased hemorrhage along the incision were apparent. Potential spaces for wound hematomas or seromas were present. No difference in the depth of tissue injury along the incision between the single port and 5-mm port was noted. The localized superficial fat necrosis was similar between both the standard and study device port sites. Figure 3 compares the single- and multi-port sites at the skin, depicting slight increases in tissue trauma apparent with discoloration of the skin (Fig. 3). At this level, you can see that the dermis remains largely without compression or significant hemorrhage with the TransEnterix single port compared with the cross section examination of the 5-mm port track where there is slightly more hemorrhage. The 5$\mathrm{mm}$ port track does not pass straight down, even though it is a relatively midline port; it actually skews slightly through the plane overlying the muscle before continuing back down. The 5-mm ports were associated with slightly more hemorrhage and dissection of some of the tissue planes than what we observed with the single-port site.

Therefore, the comparison of single port to standard laparoscopic multiport, in acute studies, showed no difference for deep tissue trauma, and slightly less creation of tissue planes and hemorrhage, as well as skin trauma with the TransEnterix single port compared with the standard 5and 10-mm laparoscopic ports.

All five of the single-port survival procedures using the TransEnterix system were completed successfully without
Fig. 3 Comparison of the skin between the single port device (A) and the standard $5 \mathrm{~mm}$ port (B) demonstrates increased epidermal sloughing and focal hemorrhage within the superficial incision in the standard 5-mm port section
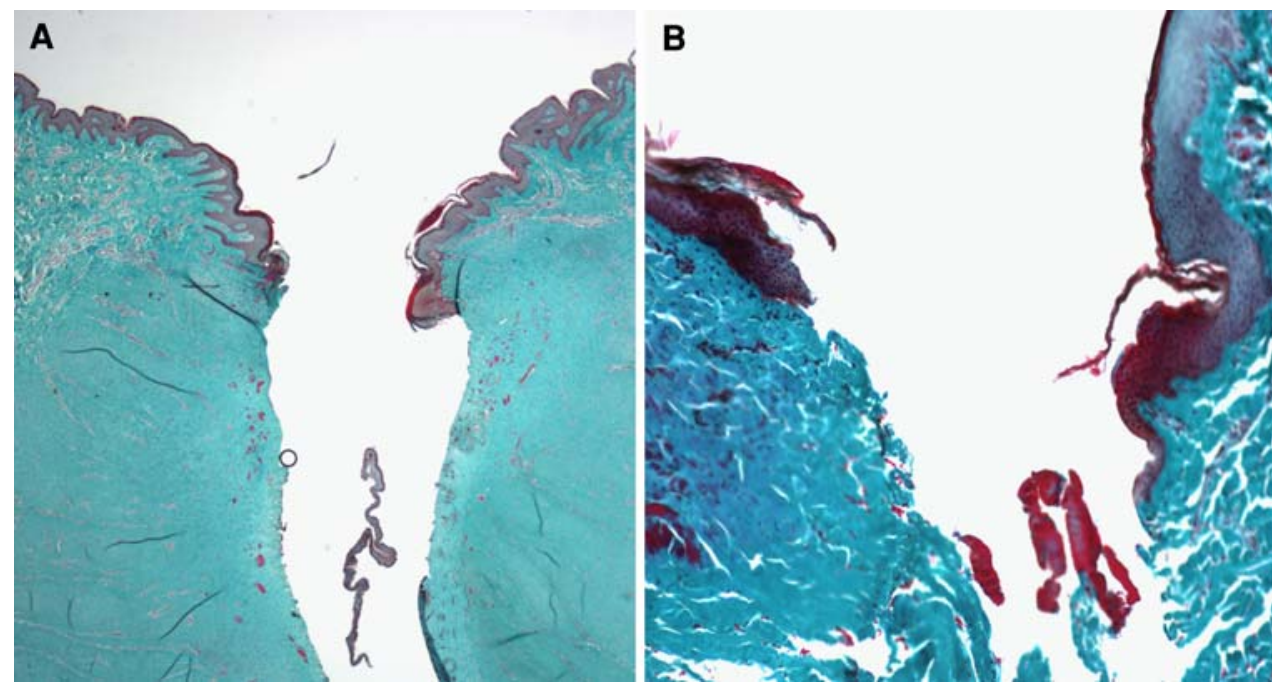


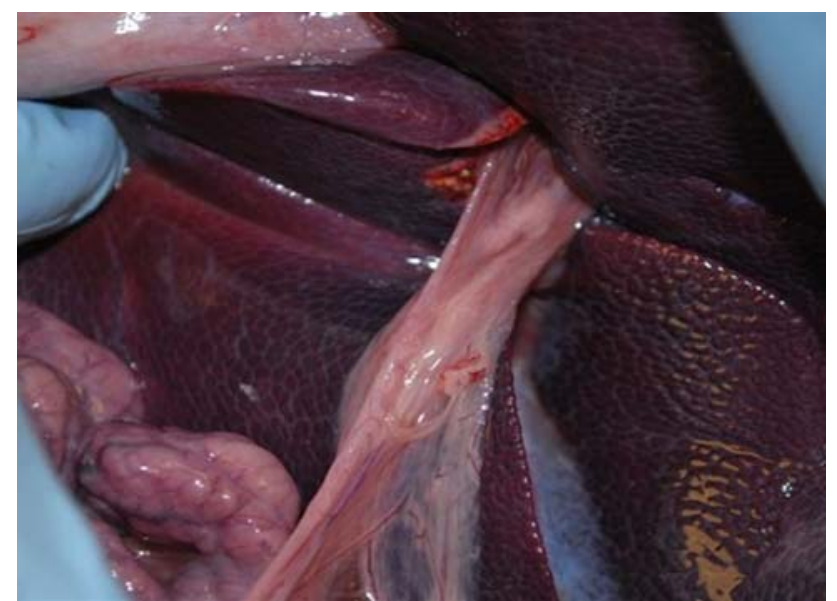

Fig. 4 Day 7 examination of liver bed revealing no biloma, hematoma or abscess, ligation clips remain intact and only mild focal omental adhesions

sequelae. Operative time averaged 39.4 (range 18-66) $\mathrm{min}$ for the procedures. At sacrifice, no complications were identified.

Seven-day survival studies of the single-port animals did not demonstrate unexpected injury or extension of nonviable tissue along the abdominal wall incision. Examination of the operative field disclosed no complications. There were mild focal omental adhesions to the gallbladder bed, as expected in single-port or multiport cholecystectomy procedure. The gross image of Fig. 4 depicts a small loop of the omentum adherent to the gallbladder bed. There were no bilomas, hematomas, or abscesses in the gallbladder bed or right upper quadrant. We observed local cautery effect, as expected from the dissection to remove the gallbladder from the swine's liver.

Peritoneal and serosal surfaces exhibited only mild focal inflammation grossly. There were some filmy peritoneal and interloop adhesions in the right upper quadrant, as occur with any laparoscopic or open cholecystectomy. There were no loculated fluid collections, abscesses, or hemorrhage present in the peritoneal cavity. The surgical clips developed for the study device single-port system all remained in place. There also was no evidence of iatrogenic visceral injury to intestines or liver, or other surrounding solid organs. Overall, the peritoneum exhibited minimal changes without evidence of unexpected injury to the peritoneal surfaces or abdominal organs.

The wound healing in the 7-day single-port swine demonstrated that inflammation was localized to the incision surface. The location and degree of inflammation was appropriate for incisional healing at 7 days. Inflammation and very early granulation tissue typical of a 7day-old wound was present along the length of the incision extending slightly into the surrounding tissue (Fig. 5). Full-thickness sections exhibited the incision

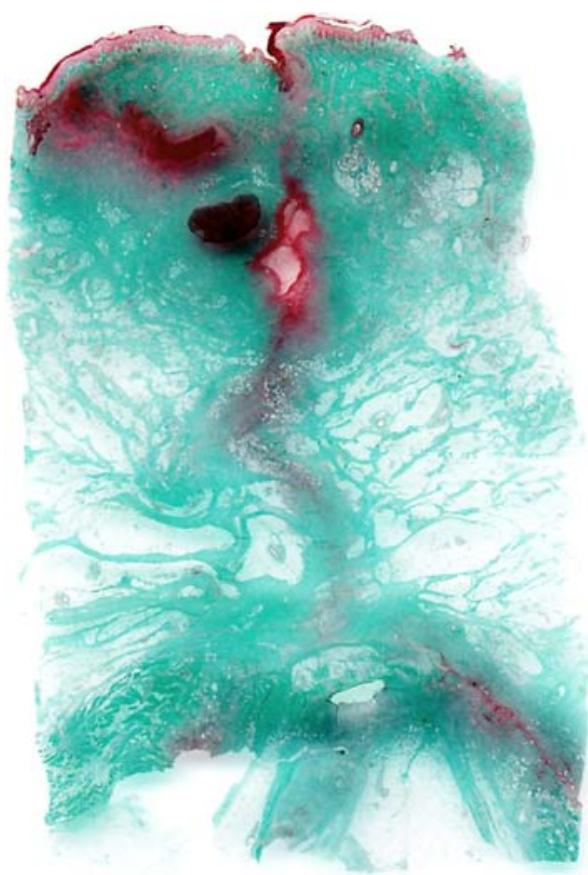

Fig. 5 Seven-day survival incision site evaluation of single port

with a slight rim of inflammation from skin to fascia (bottom). Inflammation extended slightly out from the incision surface as expected, but there was minimal tissue necrosis with no significant additional necrosis of the abdominal wall adipose, muscular, or fascial tissue. Of note, the inflammation that was present was most substantial at the level of the skin and at the peritoneal/ fascial surface, but this was associated with suture and/or staples related to wound closure. There were no significant seromas or hematomas present in the abdominal wall around the single-port sites.

Figure 6 provides a comparison of the peritoneal surface in the study device incision in acute and 7-day survival animals (Fig. 6). The image on the right side of Fig. 6 demonstrates viable fatty tissue surrounding the healing on both sides of the incision, with a normal amount of inflammation that is expected in a 7-day wound. There was no evidence of significant seroma, fat necrosis, or infection in the animals. The fatty tissue of the abdominal wall did not reveal any increased necrosis at 7 days, and as noted in Fig. 5, injury remained limited to the incision surface.

Overall, our results demonstrate minimal local tissue trauma and/or necrosis with the TransEnterix single-port surgery system compared with standard 5- and 10-mm ports used for conventional multiport laparoscopic cholecystectomy. At the 7-day time point, we did not observe any unsuspected increase in soft tissue injury or fatty necrosis. 
Fig. 6 Comparison of peritoneal surface, acute (A) versus 7 day (B), using the TransEnterix single port system reveals normal inflammation and healing without tissue necrosis
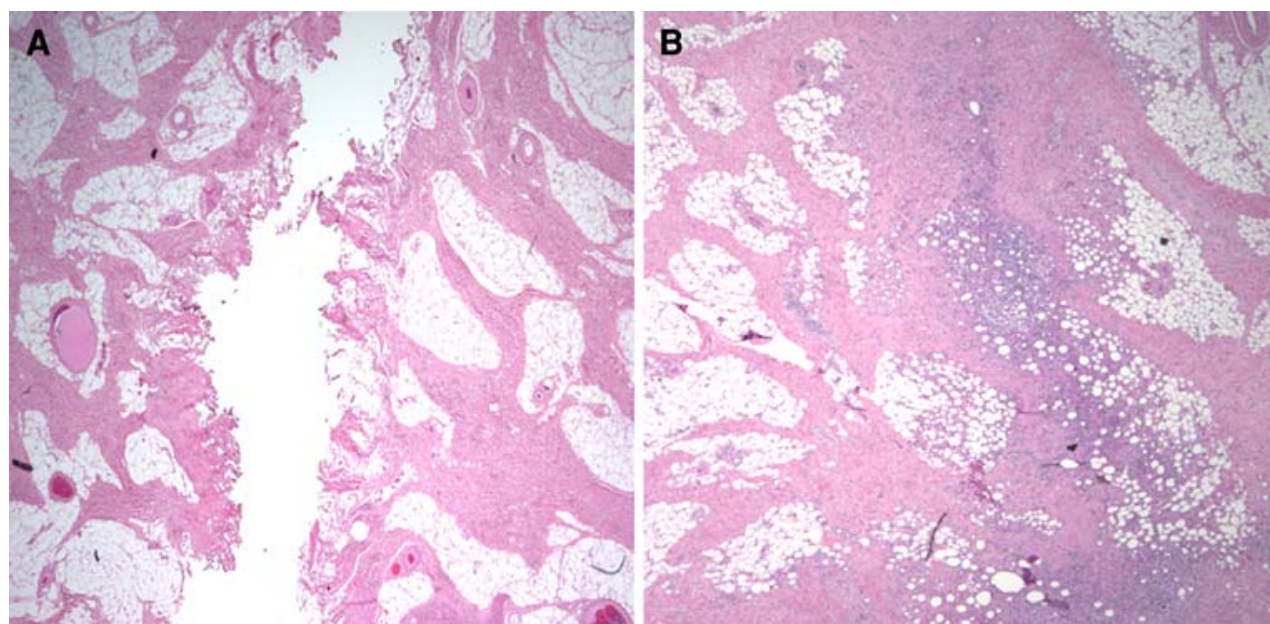

\section{Discussion}

Since the introduction of laparoscopy in the late 1980s, there have only been small advances in new and enabling techniques or advanced tools for general surgery. In the mid-portion of this decade, the first major paradigm challenge to minimally invasive surgery, known as natural orifice transluminal endoscopic surgery (NOTES), entering the abdominal cavity using one of the body's natural orifice's, rapidly became an active area for development of next-generation tools for general surgery $[9,10]$.

NOTES quickly evolved as a next frontier-a new way to think about scarless surgery, using flexible endoscopes and instruments often used for diagnostic purposes or minor procedures in the colon or upper gastrointestinal tract. Although the NOTES approach to general surgical procedures seems intriguing, there is a high level of development that must be realized in instrument designs and functionality for the NOTES technique to become widely accepted. However, the NOTES movement has triggered a wave of innovation in surgery as a whole [8]. Surgeons are challenging the surgical dogma that has been in place for many years. We have tried to develop increasingly less invasive techniques for abdominal procedures. As such, there has been a shift in general laparoscopic surgery toward single-port or single-site surgery. With the ability to eliminate visual scarring, and appealing to a patient-preferred cosmetic outcome, SPS has the potential to transform general surgery, much like the transformation from open laparotomy to laparoscopic surgery. SPS also might improve return to normal activities, based on a reduction in procedure-related infections and a potential need for fewer postoperative pain medications.

The devices available today to facilitate single-site surgery provide limited triangulation and require extensive fascial trauma at insertion. The TransEnterix SPIDER system overcomes many of these limitations. Multiple surgical instruments can be advanced and manipulated through a single port containing four working channels, thereby eliminating the need for multiple abdominal incisions-and essentially facilitating "true" SPS with one umbilical incision. Operative field visual and instrument orientation replicates current laparoscopic techniques and critical views are maintained throughout the surgical procedures. The TransEnterix's single-port SPIDER system allows surgeons to perform a number of general surgical procedures by accessing the patient's abdominal cavity and using a combination of proprietary flexible instruments and common "off-the-shelf" laparoscopic instruments. The advantage of using the system is true triangulation and simple retraction, without added operating room time or the need to tolerate uncomfortable techniques that lead to frustration and may compromise patient safety. Lower morbidity, faster recovery, and improved cosmetic results are just a few of the potential advantages of the TransEnterix system.

We have demonstrated in this study that single-port laparoscopic cholecystectomy with the TransEnterix SPIDER system results in minimal tissue trauma compared with standard four-port laparoscopic cholecystectomy. In addition, we have demonstrated that laparoscopic cholecystectomy can be accomplished with the SPIDER with reasonable operative time and minimal complications.

Some of our findings were surprising. Of particular note, we found the trauma to be slightly increased with standard 5-mm compared with 10-mm ports. Contrary to what one might believe, the smaller ports were associated with more local tissue trauma possibly due in part to the dilating placement, which is essentially through a stab incision and blunt placement with tissue more tightly surrounding the port.

We also questioned whether tissue injury may be unappreciated in the acute setting, becoming more apparent over time, as devitalized fat will undergo liquefaction necrosis and within 7 postoperative days potentially with 
wound healing issues and creation of seromas or frank wound infections. Additionally, at 7 days, we would expect early healing and inflammation to be maximized and any delayed necrosis of tissue would be identified. No additional injury was identified. Therefore, although there may be concern that a larger single-port device could require more port manipulation and local tissue injury, the current study demonstrated no increase, and possibly a reduction, of acute tissue injury compared with standard 5- and 10$\mathrm{mm}$ port sites. At 7 days survival, no additional injury was apparent and healing was appropriate without evidence of complication.

Furthermore, in comparison, the 5- and 10-mm ports exhibited increased epidermal abrasion/sloughing and mild increase in acute fascial and peritoneal injury. Standard ports also traversed more abdominal musculature and entered the peritoneal cavity off of the midline. The relatively diminished physical injury and tissue inflammation noted with the SPIDER system may provide unforeseen benefits of a true single-port platform. We propose that the diminished hemorrhage and inflammation may correlate with decreased postoperative pain and wound healing issues. Human trials will be required to adequately study this effect.

In terms of the technical aspects for performing a SPIDER cholecystectomy, many of the mechanical advantages, preferable for enabling a clear surgical dissection, are available. These include robust rigid retraction, triangulation to obtain critical view dissection, and operating through a reasonable diameter-true single port. In this study, we have shown that with these advantages, singleport cholecystectomy can be effectively and efficiently performed in a swine model. An average operative time of $39 \mathrm{~min}$ is very reasonable. Further investigation in both animal and human models will delineate the full benefit of this system.
Open Access This article is distributed under the terms of the Creative Commons Attribution Noncommercial License which permits any noncommercial use, distribution, and reproduction in any medium, provided the original author(s) and source are credited.

Disclosures Dr. A. D. Pryor is a consultant and speaker for Covidien and Olympus. She is a consultant for Gore, Transenterix and Immersion. She has an ownership interest in Transenterix and Barosense. Covidien also provides research support. Mr. J. R. Tushar is an employee of TransEnterix. Dr. L. R. DiBernardo is a consultant for Synecor, the parent company of TransEnterix.

\section{References}

1. Rane A, Dasgupta P (2009) Single-incision laparoscopic surgery. BJU Int 103(4):429-430

2. Canes D, Desai MM, Aron M et al (2008) Transumbilical singleport surgery: evolution and current status. Eur Urol 54(5):1020 1029

3. Reavis KM, Hinojosa MW, Smith BR et al (2008) Single-laparoscopic incision transabdominal surgery sleeve gastrectomy. Obes Surg 18(11):1492-1494

4. Romanelli JR, Mark L, Omotosho PA (2008) Single-port laparoscopic cholecystectomy with the TriPort system: a case report. Surg Innov 15(3):223-228

5. Tacchino R, Greco F, Matera D (2009) Single-incision laparoscopic cholecystectomy: surgery without a visible scar. Surg Endosc 23(4):896-899

6. Tracy CR, Raman JD, Cadeddu JA et al (2008) Laparoendoscopic single-site surgery in urology: where have we been and where are we heading? Nat Clin Pract Urol 5(10):561-568

7. Gumbs AA, Milone L, Sinha P et al (2009) Totally transumbilical laparoscopic cholecystectomy. J Gastrointest Surg 13(3):533-534

8. Gettman MT, Box G, Averch T et al (2008) Consensus statement on natural orifice transluminal endoscopic surgery and singleincision laparoscopic surgery: heralding a new era in urology? Eur Urol 53(6):1117-1120

9. de la Fuente SG, Demaria EJ, Reynolds JD et al (2007) New developments in surgery: Natural Orifice Transluminal Endoscopic Surgery (NOTES). Arch Surg 142(3):295-297

10. Rattner D, Kalloo A (2006) ASGE/SAGES Working Group on Natural Orifice Translumenal Endoscopic Surgery. October 2005. Surg Endosc 20(2):329-333 